\title{
Colecistectomia por Laparoscopia e por Laparotomia na Colecistite Aguda: Análise Crítica de 520 Casos
}

\author{
Laparoscopic Cholecystectomy and Open Cholecystectomy in Acute \\ Cholecystitis: Critical Analysis of 520 Cases
}

\author{
João Araújo TEIXEIRA ${ }^{1}$, Carlos RIBEIRO ${ }^{1}$, Luís M. MOREIRA², Fabiana de SOUSA로 André PINHO ${ }^{1}$, Luís GRAÇA , \\ José Costa MAIA ${ }^{1}$ \\ Acta Med Port 2014 Nov-Dec;27(6):685-691
}

\section{RESUMO}

Introdução: Apesar do cepticismo com que inicialmente foi encarada, a colecistectomia laparoscópica é hoje a técnica de eleição na colecistite aguda. Torna-se, porém, importante avaliar os seus resultados, em comparação com a colecistectomia clássica, uma vez que esta última ainda é seguida por alguns cirurgiões em determinadas situações.

Material e Métodos: No nosso estudo foram incluídos 520 doentes com colecistites agudas operados no Serviço de Cirurgia Geral do Hospital de S. João, entre 2007 e 2013, dos quais 412 (79,2\%) por laparoscopia e 108 (20,8\%) por via aberta, com uma incidência de conversão de $10,7 \%$. Procedeu-se ao estudo relativo às doenças coexistentes, leucocitose, tempo decorrido entre o diagnóstico na urgência e a cirurgia, classificação ASA, complicações intra e pós-operatórias, mortalidade, reintervenções, lesão biliar e estadia hospitalar. Os doentes convertidos foram incluídos no grupo das colecistectomias laparoscópicas. A análise estatística baseou-se em processos descritivos e a avaliação das diferenças entre grupos foi realizada com base no teste exato de Fisher, sendo considerados valores significativos para $p<0,05$.

Resultados: Colecistectomia laparoscópica versus Colecistectomia aberta: Mortalidade: $0,7 \%$ vs 3,7\% $(p=0,0369)$; Complicações per-operatórias: $3,6 \%$ vs 12,9\% ( $p=0,0006)$; Complicações pós-operatórias cirúrgicas: $7,7 \%$ vs $17,5 \%(p=0,0055)$; Pós-operatórias médicas: $4,3 \%$ vs 5,5\% ( $p=0,6077)$; Lesão da via biliar principal: $0,9 \%$ vs 1,8\% ( $p=0,6091)$; Reintervenções: $2,9 \%$ vs $5,5 \%$ ( $p=$ $0,2315)$; Internamento hospitalar inferior ou igual a quatro dias: $64,8 \%$ vs $18,5 \%(p<0,0001)$. Na colecistectomia laparoscópica houve $10,7 \%$ de conversões: nas precoces (intervenções realizadas antes das 96 h após o diagnóstico na urgência) esta taxa foi de $8,8 \%$ e nas tardias (após aquele período de tempo mas no mesmo internamento) de 13,7\% $(p=0,1425)$; Complicações nos doentes convertidos vs não convertidos: nas cirúrgicas $20,4 \%$ vs $6,2 \%(p=0,0034)$ e nas médicas $6,8 \%$ vs $4,1 \%(p=0,4484)$. As causas de conversão foram condicionadas por complicações cirúrgicas (lesões biliares, lacerações entéricas, perfurações vesiculares com a disseminação de cálculos), intolerância ao pneumoperitoneo, indefinição do pedículo biliar e escoliose.

Discussão: Há poucas investigações relativas à comparação da colecistectomia laparoscópica vs colecistectomia aberta nos doentes com colecistectomia aberta, correspondendo a maior parte delas a estudos multicêntricos. Por esta razão, julgamos de interesse proceder a uma análise inerente a 520 operados com aquela doença no Serviço de Cirurgia Geral do Hospital de S. João dos quais 412 por colecistectomia laparoscópica e 108 por colecistectomia aberta. Verificamos na colecistectomia laparoscópica melhores resultados do que na colecistectomia aberta no que se refere à mortalidade, complicações per e pós-operatórias cirúrgicas e estadia hospitalar. A incidência da via biliar principal, complicações médicas e reintervenções, embora menos evidentes na colecistectomia laparoscópica, não se revelaram com significado estatístico. Merece referência o maior número de complicações no grupo das colecistectomias laparoscópicas convertidas do que naquelas em que tal não foi necessário confirmando-se, assim, o já referido em estudos multicêntricos citados na literatura. Este facto levanta a necessidade de, mediante complicações ocorridas durante a colecistectomia laparoscópica, não se proceder à conversão tardiamente. A análise do presente estudo valoriza, assim, devidamente a colecistectomia laparoscópica na cirurgia dos doentes com colecistite aguda.

Conclusão: Os resultados obtidos justificam a frequência com que a colecistectomia laparoscópica é realizada na colecistite aguda, em comparação com a via aberta, ocupando cada vez mais, um lugar primordial, no tratamento desta doença.

Palavras-chave: Colecistectomia Laparoscópica; Colecistectomia; Colecistite.

\section{ABSTRACT}

Introduction: Despite the skepticism with which it was initially seen, laparoscopic cholecystectomy is now the technique of choice for acute cholecystitis. It is, however, important to evaluate the results in comparison with classic cholecystectomy, since the latter is still used by some surgeons in certain situations.

Material and Methods: Our research corresponds to the analysis of 520 patients operated on for acute cholecystitis performed in the department of surgery at the São João Hospital in Oporto - 412 (79.2\%) laparoscopic cholecystectomies and 108 (20.8\%) open cholecystectomies - from 2007 to 2013 . We evaluated comorbidities, leukocytosis, time between diagnosis and surgery, ASA, per and postoperative complications, mortality, reoperations, lesion of main bile duct, conversion rate and hospital stay, in order to compare these two techniques. The conversion group was included in laparoscopic cholecystectomy. Statistical analysis was based on descriptive statistic procedures and the evaluation of contrast between groups was based on Fishers' exact test. Significant values were considered for $p<0.05$.

Results: Laparoscopic Cholecystectomy versus Open Cholecystectomy: Mortality: $0.7 \%$ vs 3,7\% ( $p=0.0369$ ); Peroperative complications: $3.6 \%$ vs $12.9 \%(p=0.0006)$; Surgical postoperative complications: $7.7 \%$ vs $17.5 \%(p=0.0055)$; Medical postoperative complications: $4.3 \%$ vs $5.5 \%(p=0.6077)$; Lesion of the main bile duct: $0.9 \%$ vs $1.8 \%(p=0.6091)$; Reoperation: $2.9 \%$ vs $5.5 \%$ ( $p=$ 0.2315); Hospital stay up to 4 days after surgery: $64.8 \%$ vs $18.5 \%(p<0.001)$. The convertion rate was of $10.7 \%: 8.8 \%$ in early surgery

1. Serviço de Cirurgia. Faculdade de Medicina. Universidade do Porto/Hospital de S. João. Porto. Portugal.

2. Gabinete de Estudos Metodológicos e Tratamento de Dados. Escola Superior de Saúde de Vila Nova de Gaia. Instituto Piaget. Gaia. Portugal.

Recebido: 12 de Fevereiro de 2014 - Aceite: 02 de Outubro de 2014 | Copyright @ Ordem dos Médicos 2014 
(before 4 days after de diagnosis) and $13.7 \%$ in the late surgery (after this time but in the same stay) $(p=0.1425)$. Multiple causes led to convertion: surgical complications (biliary lesions, iatrogenic lesion of the small bowel, perfurations of the gallbladder with spillage of stones); complications during the pneumoperitoneum, unclear anatomy and scoliosis. Postoperative complications in laparoscopic cholecystectomies converted group vs non-converted: surgical $20.4 \%$ vs $6.2 \%(p=0.0034)$ and medical $6.8 \%$ vs $4.1 \%(p=0.4484)$.

Discussion: There are few investigations concerning the comparison of laparoscopic cholecystectomy vs acute cholecystitis in patients with acute cholecystitis, corresponding mostly to multicenter studies. For this reason, we carry out an analysis inherent to 520 patients operated on with that disease in the surgery department of Hospital S. João in Oporto of which 412 were by laparoscopic cholecystectomy and 108 by acute cholecystitis. We found better results in laparoscopic cholecystectomy than in acute cholecystitis with respect to mortality, per and post-operative surgical complications and hospital stay. The incidence of main bile duct injury, medical complications and reoperations, although less evident in laparoscopic cholecystectomy, were not statistically significant. There were more complications in the group of laparoscopic cholecystectomy converted than in those where it was not be necessary the conversion. This raises the need, in complications during the laparoscopic cholecystectomy, not to perform the conversion too late. The analysis of this study, therefore, properly values laparoscopic cholecystectomy in the surgery of patients with acute cholecystitis.

Conclusion: The results justify the frequency with which laparoscopic cholecystectomy is performed in acute cholecystitis, in comparison to open surgery, thus taking an increasingly prominent place in the treatment of this disease.

Keywords: Cholecystectomy, Laparoscopic; Cholecystectomy; Cholecystitis.

\section{INTRODUÇÃO}

A abordagem laparoscópica foi inicialmente considerada como uma contraindicação na cirurgia da colecistite aguda. ${ }^{1}$ A partir de meados de 1990 , começou a ser a técnica de eleição naquela doença. ${ }^{2,3}$ Contudo, ainda hoje alguns autores continuam, em determinadas circunstâncias, a recorrer à colecistectomia por via aberta. ${ }^{4}$

\section{MATERIAL E MÉTODOS}

De janeiro de 2007 a outubro de 2013 foram operados 520 doentes com colecistite aguda no Serviço de Cirurgia Geral do Hospital de S. João do Porto, internados pelo Serviço de Urgência daquele Hospital.

Realizaram-se $412(79,2 \%)$ colecistectomias laparoscópicas $(C L)$ e $108(20,8 \%)$ colecistectomias abertas (CA). Neste estudo, foram excluídas as colecistostomias. A opção entre os dois tipos de cirurgia foi efetuada em função da experiência do cirurgião em cirurgia mini-invasiva, da existência de alterações sistémicas graves e de peritonite biliar, tendo este último grupo sido incluído nas colecistectomias por via aberta. O grupo de indivíduos convertidos foi considerado na série das colecistectomias laparoscópicas.

O diagnóstico foi realizado em função de elementos clínicos, analíticos e imagiológicos (ecografia). Em todos os casos houve confirmação histológica.

A cirurgia foi realizada mediante a ineficácia da terapêutica médica devidamente instituída.

Em 16 casos, manifestou-se estado de choque, em 11 $(2,6 \%) \mathrm{CL}$ e em cinco $(4,6 \%) \mathrm{CA}$; em todos procedeu-se, antes da cirurgia, à conveniente correção das alterações hemodinâmicas; houve $16(3,8 \%)$ icterícias na $\mathrm{CL}$ e sete $(6,4 \%)$ na CA, por litíase canalicular. Registaram-se 23 litíases coledócicas diagnosticadas no decurso da ecografia. Estes doentes foram transferidos da urgência para o serviço de cirurgia normal e operados no decurso do mesmo internamento. Em 16, procedeu-se à extração dos cálculos, por via endoscópica, três a quatro dias antes da CL; nos outros sete, realizou-se o tratamento da litíase coledócica durante a CA.

A idade média ( \pm desvio padrão) dos pacientes por tipo de intervenção foi de 55,24 anos $( \pm 16,8)$ na $C L$ e 70,55 anos $( \pm 14,7)$ na CA.

A técnica cirúrgica foi relatada em publicação anterior. ${ }^{5}$
A análise estatística baseou-se em processos descritivos e a avaliação das diferenças entre grupos teve por base o teste exato de Fisher, sendo considerados valores significativos para $p<0,05$.

\section{RESULTADOS}

Apresentamos abaixo o número de casos intervencionados por via laparoscópica (CL) e por via aberta (CA), em função do género, doenças coexistentes, leucocitose, tempo decorrido entre o diagnóstico na urgência e a cirurgia, ASA, complicações intra e pós-operatórias, mortalidade, reintervenções, lesão biliar e estadia hospitalar.

Género: Homens: CL 204 (49,5\%) e CA 46 (42,5\%); $p=0,2340$; Mulheres: CL $208(50,4 \%)$ e CA $62(57,4 \%)$; $p=0,2340$.

Leucocitose superior a 12.000.000/m/ ${ }^{3}:$ CL 267 (64,8\%) e CA $89(82,4 \%), p=0,0004$.

Tempo decorrido entre o diagnóstico na urgência e a cirurgia (que decorreu no mesmo internamento):

- CL: Inferior ou igual a quatro dias $250(60,6 \%)$, Superior a quatro dias $162(39,3 \%), p<0,001$;

- CA: Inferior ou igual a quatro dias $84(77,7 \%)$, Superior a quatro dias $24(22,2 \%) p<0,001$.

ASA III e IV: nestes grupos incluiam-se 85 (20,6\%) operados por via laparoscópica e $40(37,0 \%)$ por via aberta; $p=0,0006$ (Tabela 1).

Complicações intra-operatórias: manifestaram-se 15 $(3,6 \%)$ na CL e $14(12,9 \%)$ na CA ( $p=0,0006)$. Incluem-se nas $C L$ as complicações responsáveis por conversões (Tabela 2).

Ocorreram três lesões da VBP, dois na CL e um na CA. Verificaram-se, ainda, seis lesões do canal cístico, três na CL (dois no decurso da disseção da fístula colecisto-duodenal com fenómenos inflamatórios intensos, sendo a outra consequente à extração de volumoso cálculo em vesícula escleroatrófica) e três na CA. Estas últimas foram ocasionadas, durante a disseção colecisto-cística para a realização da colangiografia per-operatória. Houve seis hemorragias, três por lesão da artéria cística (dois na CL e um na $\mathrm{CA}$ ) e três no decurso da disseção vesicular do leito hepático (dois na CL e um na CA). As perfurações vesiculares registaram-se sempre em colecistites gangrenosas, 
Tabela 1 - Doenças coexistentes

\begin{tabular}{lccc}
\hline & Doenças Coexistentes & & \\
\hline & CL & CA & p \\
Diabetes & $57(13,8 \%)$ & $25(23,1 \%)$ & 0,0253 \\
Cardiovasculares & $117(28,3 \%)$ & $49(45,3 \%)$ & 0,011 \\
Doença pulmonar obstrutiva & $21(5,1 \%)$ & $6(5,6 \%)$ & 0,8097 \\
Insuficiência renal crónica & $17(4,1 \%)$ & $10(9,2 \%)$ & 0,0478 \\
\hline
\end{tabular}

Tabela 2 - Complicações per-operatórias

\begin{tabular}{lcc}
\hline \multicolumn{1}{l}{ Complicações per-operatórias } & $\boldsymbol{p}=\mathbf{0 , 0 0 0 6}$ \\
\hline & CL (15) & CA (14) \\
\hline Lesão VBP & 2 & 1 \\
Hemorragias & 4 & 2 \\
Lesão do canal cístico & 3 & 3 \\
Perfuração vesiculares & 5 & 6 \\
Lesão iatrogénica do intestino delgado & 1 & 2 \\
\hline
\end{tabular}

acompanhando-se de larga disseminação abdominal de cálculos. A lesão iatrogénica do intestino delgado, na $\mathrm{CL}$, foi consecutiva à introdução de um trocarte para a realização do pneumoperitoneu. Registaram-se, ainda, duas lesões entéricas na CA, durante a disseção de duas fístulas colecisto-duodenais.

\section{Complicações pós-operatórias:}

a) Cirúrgicas: a seguir à CL 32 (7,7\%) e na CA 19 (17,5\%); $p=0,0055$ (Tabela 3)

As 15 fístulas biliares foram ocasionadas por: três lesões da VBP (dois CL e um CA), nove bilomas (cinco CL e quatro $C A$ ), duas deiscências do canal cístico (CL) e ainda uma deiscência de hepático-jejunostomia em CL convertida por lesão da VBP (este último doente corresponde a um dos óbitos). As três lesões da VBP foram corrigidas por anastomose bilio-digestivo. Os bilomas trataram-se por drenagem percutânea orientada por ecografia em seis casos (três CL e três CA) e no decurso de CPRE noutros três (duas CL e uma CA). Houve 18 abcessos abdominais (13 nas $\mathrm{CL}$ e cinco nas $\mathrm{CA}$ ); apenas em duas (CL) foi necessário reintervir cirurgicamente, tendo os restantes sido drenados por via percutânea sob controlo ecográfico. Verificaram-se quatro hemorragias, duas nas CL (uma consecutiva à lesão iatrogénica do baço e outra a laceração do leito hepático) e duas nas CA (lesão da artéria cística). A amputação do pé diabético verificou-se num doente em que se instalou quadro séptico a seguir à cirurgia. Como já foi referido, nestas

Tabela 3 - Complicações pós-operatórias cirúrgicas

\begin{tabular}{|c|c|c|}
\hline Complicações pós-operatórias: Cirúrgicas & $p=0,0055$ & \\
\hline & CL (32) & CA (19) \\
\hline Abcessos abdominais & $13(2 *)$ & 5 \\
\hline Infeções ao nível da parede & $6(5 *)$ & 2 \\
\hline Eventração & 0 & 3 \\
\hline Hemoperitoneu & 2 & 2 \\
\hline Amputação de pé diabético & 1 & 0 \\
\hline Fístulas biliares: & $10(2 *)$ & 5 \\
\hline - Lesões da VBP & 2 & 1 \\
\hline - Deiscência do coto cístico & 2 & -- \\
\hline - Deiscência da anastomose hepato-jejunal & $1(* *)$ & --- \\
\hline - Bilomas & 5 & 4 \\
\hline Perfuração cólica & 0 & 1 \\
\hline Oclusão & 0 & 1 \\
\hline
\end{tabular}

$\left(^{\star}\right)$ doentes convertidos; ${ }^{(* *}$ lesão da VBP em CL convertida. 
complicações, incluíram-se os doentes das CL convertidas. b) Médicas: No pós-operatório manifestaram-se 24 complicações de foro médico: $18(4,4 \%)$ na CL e seis $(5,5 \%)$ a seguir à $\mathrm{CA}(p=0,607)$ (Tabela 4$)$.

A maior parte destas complicações manifestaram-se em indivíduos com idade superior a 70 anos (12 na CL e cinco na CA). Num caso, instalou-se falência multiorgânica correspondente a complicações cardiovasculares e renais.

Mortalidade operatória: Houve sete óbitos, sendo três $(0,7 \%)$ na $C L$ e quatro na $C A(3,7 \%) ; p=0,0369$. As causas de morte na CL foram: um AVC, uma peritonite com choque séptico e uma lesão da via biliar principal. Na CA: um choque séptico consecutivo a abcesso abdominal com complicações respiratórias, uma lesão iatrogénica do cólon, uma falência multiorgânica (com insuficiência renal grave), uma hemorragia do leito vesicular em doente com lesão da VBP corrigida no per-operatório. Nos sete óbitos, cinco correspondiam a colecistites gangrenosas (três na CL e duas na CA).

Lesão da via biliar principal: Registaram-se seis lesões da VBP, quatro $(0,9 \%)$ na $C L$ e dois $(1,8 \%)$ na $C A$ $(p=0,6091)$; sendo três diagnosticadas no per-operatório (duas $\mathrm{CL}$ e uma $\mathrm{CA}$ ) e três no pós-operatório imediato (duas CL e uma CA); todas elas se incluíam no grupo II da classificação de Bismuth; duas foram ocasionadas por feridas laterais do colédoco, verificando-se nas outras qua- tro, uma secção transversal daquele canal; foram tratadas por anastomose termino-terminal (uma CL e uma CA) e por hepático-jejunostomia (duas CL e duas CA).

Conversões: Houve um total de 44 conversões $(10,7 \%)$. Nos indivíduos operados nos primeiros quatro dias a seguir ao diagnóstico (efetuado na urgência) essa taxa foi de $8,8 \%$ e nos que foram operados após aquele período de tempo, mas, no mesmo internamento, ocorreu em $13,7 \%(p=0,1425)$.

As causas responsáveis foram: 10 colecistites gangrenosas, com disseminação de cálculos, 27 indefinições de pedículo biliar (duas fístulas colecisto-duodenais), duas lesões da VBP, duas lesões do canal cístico, uma lesão iatrogénica do delgado, uma escoliose, uma intolerância ao pneumoperitoneu. A incidência em relação ao género foi de $8,8 \%$ nos homens e $12,5 \%$ nas mulheres ( $p=0,2661)$. No que respeita à idade, essa taxa foi de $16,5 \%$ nos indivíduos com mais de 65 anos e de $7,6 \%$ nos grupos mais jovens $(p=0,106)$. No grupo convertido registaram-se as seguintes complicações em relação aos doentes não convertidos: nas cirúrgicas $20,4 \%$ vs $6,2 \%(p=0,0034)$ e nas médicas $6,8 \%$ vs $4,1 \%(p=0,4484)$.

Reintervenções: Realizaram-se $12(2,9 \%)$ na CL e seis $(5,5 \%)$ na $C A(p=0,2315)$ em função das seguintes causas (Tabela 5).

Estas reintervenções consistiram em oito laparotomias

Tabela 4 - Complicações pós-operatórias médicas

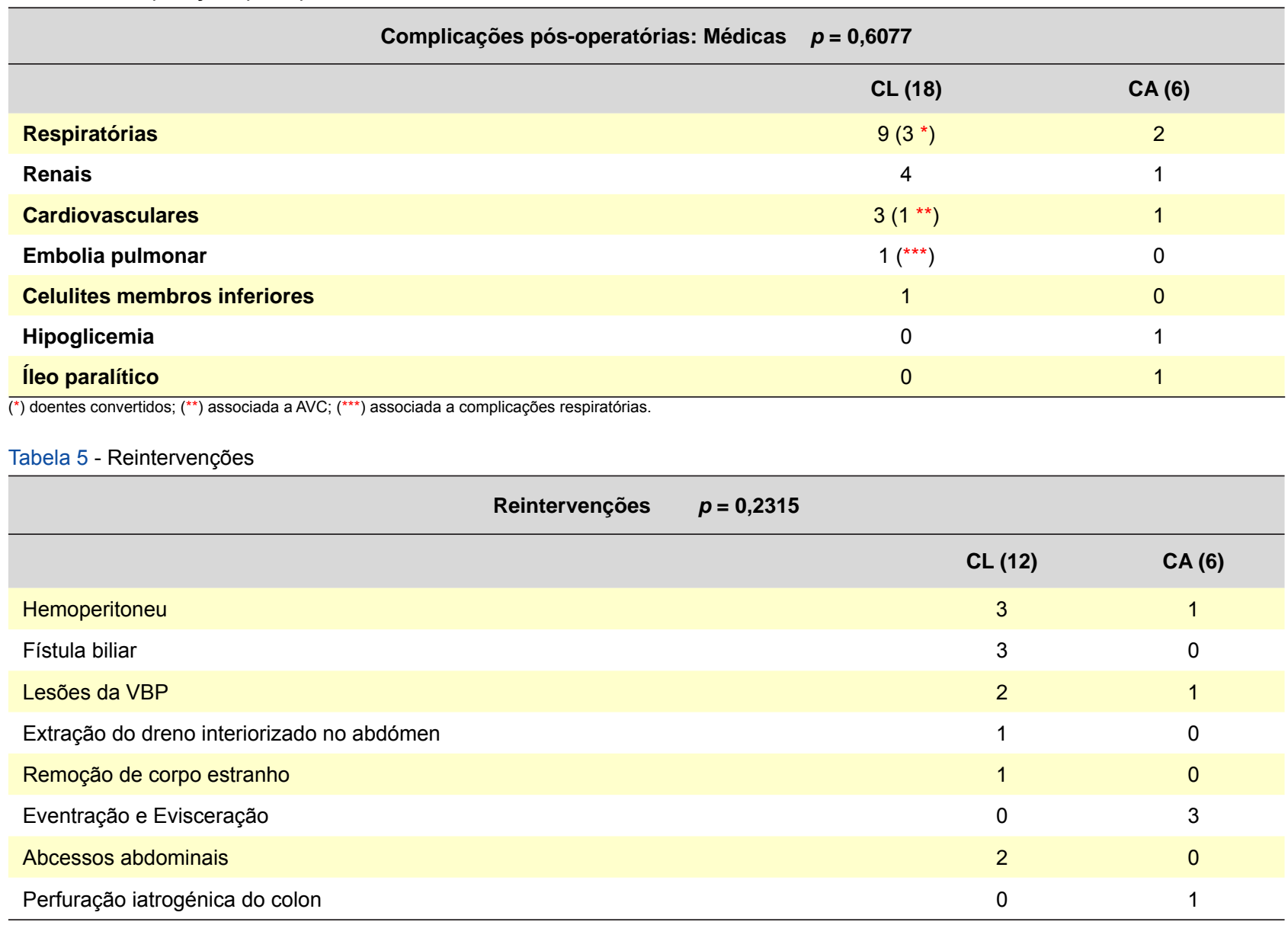


Tabela 6 - Análise global

\begin{tabular}{|c|c|c|c|c|c|}
\hline & \multicolumn{2}{|c|}{ CL } & \multicolumn{2}{|c|}{ CA } & \multirow[b]{2}{*}{$p$ - value } \\
\hline & $n$ & $\%$ & $n$ & $\%$ & \\
\hline Complicações intraoperatórias & 15 & 3,6 & 14 & 12,9 & $0,0006^{* *}$ \\
\hline Complicações pós-operatórias: globais & 50 & 12,1 & 25 & 23,1 & $0,0055^{\star \star}$ \\
\hline Complicações pós-operatórias: cirúrgicas & 32 & 7,7 & 19 & 17,5 & $0,0055^{\star *}$ \\
\hline Complicações pós-operatórias médicas & 18 & 4,3 & 6 & 5,5 & 0,6077 \\
\hline Reintervenções & 12 & 2,9 & 6 & 5,5 & 0,2315 \\
\hline Lesão VBP & 4 & 0,9 & 2 & 1,8 & 0,6091 \\
\hline Mortalidade & 3 & 0,7 & 4 & 3,70 & $0,0369^{*}$ \\
\hline Estadia Hospitalar $<4$ dias & 267 & 64,8 & 20 & 18,5 & $<0,0001^{* *}$ \\
\hline
\end{tabular}

${ }^{* \star} p<0,01 ;{ }^{*} p<0,05$.

(sete nas CL e uma na CA), uma esplenectomia $(C L)$, três correções de deiscência parietais (três $\mathrm{CA}$ ), três anastomoses bilio-digestivas (duas CL e uma CA) e uma colostomia. A remoção do dreno pós-operatório interiorizado no abdómen, o corpo estranho, e um abcesso foram operados por laparoscopia. Nas sete laparotomias da CL está incluído o caso da deiscência hépato-jejuonal em CL convertida por lesão per-operatória da VBP em que apenas se pôde realizar drenagem biliar.

Estadia hospitalar: Foi inferior ou igual a quatro dias em 171 casos $(41,5 \%)$ na CL e em $20(18,5 \%)$ na $C A$ $(p<0,001)$.

\section{DISCUSSÃO}

A Colecistectomia Laparoscópica, que foi considerada inicialmente uma contraindicação na colecistite aguda, com o decorrer do tempo veio a ser o gold standard do tratamento cirúrgico desta doença. ${ }^{6,7}$ Porém, ainda hoje, alguns autores em determinadas circunstâncias consideram lícito recorrer à via aberta. Assim, Navez' refere: 'Although laparoscopic cholecystectomy is considered a standard treatment for acute cholecystitis, an open approach is still a valid option for more advanced disease'.

Julgamos de interesse analisar a estatística do nosso Serviço correspondente a 520 daquelas intervenções, tanto mais que a maior parte das séries referidas correspondem a estudos multicêntricos, ${ }^{7,8}$ incluindo cirurgias realizadas por diversos centros cirúrgicos. A nossa presente investigação tem a vantagem de constituir uma série homogénea, com cirurgiões do mesmo Serviço e técnicas estandardizadas.

$\mathrm{Em}$ todas as investigações recentemente publicadas verifica-se o predomínio da CL vs CA: $93,2 \%$ vs $6,8 \%{ }^{7} 85 \%$ vs $15 \%{ }^{9}$ e $84,4 \%$ vs $14,6 \%,{ }^{10}$ sendo igualmente, desde há muito, a intervenção mais utilizada no nosso Serviço.

Em relação à nossa última publicação sobre este assunto, ${ }^{6}$ verificou-se um aumento da CL $(67,1 \%$ vs $84,8 \%$; $p<0,0001)$ e uma diminuição da CA $(25,7 \%$ vs $15,2 \% ; p=$ $0,0034)$, em função da crescente diferenciação dos nossos cirurgiões mais jovens em técnicas mini-invasivas.
A análise global dos nossos resultados (Tabela 6) confirma as vantagens da CL vs CA no que diz respeito à mortalidade, às complicações per e pós-operatórias cirúrgicas e à estadia hospitalar. A lesão da VBP foi inferior na CL em relação à $\mathrm{CA}(0,9 \%$ vs $1,8 \%)$, embora sem valor significativo; correspondeu ao geralmente referido na literatura $(\mathrm{CL}$ vs CA): $0,2 \%$ vs $1 \% ;{ }^{9} 1,08 \%$ vs $2,7 .^{7}$ Das seis lesões da VBP, três foram reconhecidas durante o ato cirúrgico (duas $\mathrm{CL}$ e uma $\mathrm{CA}$ ) e três no pós-operatório (duas $\mathrm{CL}$ e uma $\mathrm{CA}$ ). Destas últimas três, em duas (uma $\mathrm{CL}$ e uma $\mathrm{CA}$ ) não foi possível realizar a colangiografia per-operatória por intensos fenómenos inflamatórios inerentes à junção colecisto-cística; noutro caso (CL), a lesão verificou-se durante o ato cirúrgico, apesar de ser efetuada a colangiografia.

Aliás, não há unanimidade sobre o valor daquele exame no decurso da colecistectomia. Alguns consideram-no imprescindível, ${ }^{13}$ valorizando-o igualmente no diagnóstico per-operatório da litíase coledócica coexistente com a colecistite aguda; ${ }^{7}$ outros apenas o realizam de modo seletivo. ${ }^{14}$

Pela nossa parte, recorremos a ele sempre que se torna conveniente a definição do pedículo biliar, embora as alterações inflamatórias por vezes aí existentes nem sempre facilitem a sua realização.

Torna-se importante referir que o sucessivo adestramento técnico dos diversos cirurgiões em cirurgia laparoscópica tem vindo a diminuir consideravelmente esta complicação.

A correção daquelas lesões consistiu em quatro hepático-jejunoestomias (duas CL e duas CA), duas anastomoses coledócicas termino-terminais; todas foram incluídas no grupo 2 na classificação de Bismuth.

Para além das lesões da VBP acima citadas, merecem referência as fístulas biliares ocasionadas por deiscência do canal cístico o que é bem demonstrativo dos cuidados com que se deve rodear a laqueação deste canal. Igualmente é de destacar a presença de fístulas biliares ocasionadas por bilomas, provavelmente na dependência de canais biliares acessórios devidamente assinalada na literatura. ${ }^{7}$ 
Nas hemorragias, para além das lesões da artéria cística (duas CA) devem ser valorizadas as dependentes da dissecções da vesícula do leito vesicular e ainda a lesão iatrogénica do baço.

Registamos uma conversão de 10,3\%, valor semeIhante ou mesmo inferior ao diversamente relatado: $10 \%$; $19,7 \%{ }^{10} ; 10,7 \%$ e $32 \%$ (correspondendo estes últimos valores a casos particularmente graves). ${ }^{7}$

Têm sido referidos maior incidência de conversão no sexo masculino ${ }^{7}$ e no grupo de idosos ${ }^{14}$; no nosso estudo verificamos conversão mais elevada nos homens e nos indivíduos com mais de 65 anos, embora estes valores não fossem estatisticamente significativos.

Assinalamos maior incidência de complicações cirúrgicas no grupo de CL convertido do que naquele em que tal não foi necessário: $20,4 \%$ vs $6,2 \%(p=0,0034)$. Este facto deve ser suficientemente valorizado tanto mais que já foi anteriormente assinalado por $\mathrm{Navez}^{7}$; levanta a reflexão sobre a oportunidade de mediante CL complexas, não se proceder tardiamente à conversão.

O tempo decorrido entre a cirurgia e o diagnóstico inicial (considerando-se o limite de quatro dias para a denominação de cirurgia precoce), tem sido valorizado diferentemente, de acordo com vários autores. A CL precoce proporcionaria menor conversão em várias publicações. ${ }^{15-17}$ Outros referem opinião contrária, não atribuindo qualquer diferença na cirurgia precoce comparada com a tardia. ${ }^{10,18,19}$

Na presente investigação, a conversão correspondente à CL precoce foi de $8,3 \%$ e de $13,7 \%$ na tardia (após quatro dias). No entanto, a análise estatística destes parâmetros não foi significativa. As causas de conversão foram condicionadas por complicações cirúrgicas (lesões biliares, lacerações entéricas, perfurações vesiculares em colecistites gangrenosas com disseminação abdominal de cálculos), intolerância ao pneumoperitoneo, indefinição do pedículo biliar e escoliose.

\section{REFERÊNCIAS}

1. Cuschieri A, Berci G. Laparoscopic biliary surgery. Oxford: Blackwell Scientific Publications; 1991.

2. Eldar S, Sabo E, Nash E, Abrahamson J, Matter I. Laparoscopic cholecystectomy for the various types of gallbladder inflammation: a prospective trial. Surg Laparosc Endosc. 1998;8:200-7.

3. Teixeira JP, Saraiva AC, Cabral AC, Barros H, Reis JR, Teixeira A. Laparoscopie ou laparotomie dans la cholécystite aiguë. Chirurgie. 1999;124:529-35.

4. Bernstein D, Soeffing J, Daoud YJ, Fredin J, Kravet SJ. The obscure gallbladder. Am J Med. 2007;120:675-7.

5. Teixeira JP. How to perform a safe laparoscopic cholecystectomy.In: Araújo Teixeira JP,editor. Minimally invasive abdominal surgery, Lisboa: Instituto Piaget; 2010. p.67-82.

6. Teixeira JP, Ribeiro C, Pinto A, Goulart A, Maia JC, Pimenta A. Surgical management of acute cholecystitis (experience of 249 cases). Hepatogastroenterology. 2011;58:281-4.

7. Navez B, Ungureanu F, Michiels M, Claeys D, Muysoms F, Hubert C, et al. Surgical management of acute cholecystitis: results of a 2-year prospective multicenter survey in Belgium. Surg Endosc. 2012;26:2436-45.

8. Borzellino G, Sauerland S, Minicozzi AM, Verlato G, Pietrantonj CD, Manzoni G, et al. Laparoscopic cholecystectomy for severe acute cholecystitis. A meta-analysis of results. Surg Endosc. 2008;22:8-15.
A mortalidade por nós referida correspondeu a sete casos: três $(0,7 \%)$ em CL e quatro em CA, constituindo valores aproximados aos citados noutros estudos: 0,4\% vs 4\%; 9 $0,5 \%$ vs $5,4 \%{ }^{7}$; de registar que naqueles óbitos, dois foram incluídos duas lesões da VBP em doentes com colecistites gangrenosas.

A estadia hospitalar foi inferior na $C L$ do que na $C A$, como aliás seria de esperar. $\mathrm{Na} \mathrm{CL} 64,8 \%$ dos indivíduos tiveram uma estadia hospitalar inferior a quatro dias, enquanto na CA essa taxa foi de 18,5\% ( $p<0,0001)$.

Pretendemos insistir nas enormes vantagens da CL sobre a CA, no tratamento da colecistite aguda. Contudo, o seu uso deve estar condicionado por vários fatores, devidamente assinalados na literatura, como experiência do cirurgião nesta cirurgia e alterações sistémicas multiorgânicas do operado. ${ }^{10,20,21}$

\section{CONCLUSÃO}

A análise dos nossos resultados justifica a frequência com que a colecistectomia laparoscópica é utilizada no nosso Serviço na cirurgia da colecistite aguda confirmando, assim, a importância cada vez maior da cirurgia mini-invasiva no tratamento daquela doença.

\section{OBSERVAÇÕES}

Parte deste estudo foi apresentado em 07.fev.2014 na

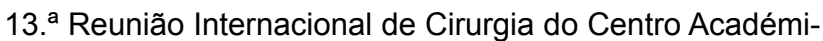
co de Medicina da Faculdade de Medicina da Universidade de Lisboa.

\section{CONFLITOS DE INTERESSE}

Os autores declaram que não houve conflito de interesse nesta investigação.

\section{FONTES DE FINANCIAMENTO}

Os autores declaram que não houve financiamentos nesta investigação.

9. Csikesz N, Ricciardi R, Tseng JF, Shah SA. Current status of surgical management of acute cholecystitis in the United States. World J Surg. 2008;32:2230-6.

10. Degrate L, Ciravegna AL, Luperto M, Guaglio M, Garancini M, Maternini $\mathrm{M}$, et al. Acute cholecystitis: the golden 72-h period is not a strict limit to perform early cholecystectomy. Results from 316 consecutive patients. Langenbeck's Arch Surg. 2013;398:1129-36.

11. Berci G, Hunter J, Morgenstern L, Arregui M, Brunt M, Carroll B, et al. Laparoscopic cholecystectomy: first, do no harm; second, take care of bile duct stones. Surg Endosc. 2013;27:1051-4.

12. Hamad MA, Nada AA, Abdel-Atty ME, Kawashti AS. Major biliary complications in 2,714 cases of laparoscopic cholecystectomy without intraoperative cholangiography: a multicenter retrospective study. Surg Endosc. 2011;25:3747-51.

13. Suliman E, Palade R. Laparoscopic cholecystectomy for treating acute cholecystitis - possibilities and limitations. Chirurgia. 2013;108:32-7.

14. Kirshtein B, Bayme M, Bolotin A, Mizrahi S, Lantsberg L. Laparoscopic cholecystectomy for acute cholecystitis in the elderly: is it safe? Surg Laparosc Endosc Percutan Tech. 2008;18:334-9.

15. Teixeira JP, Saraiva AC, Cabral AC, Barros H, Reis JR, Teixeira A. Conversion factors in laparoscopic cholecystectomy for acute cholecystitis. Hepatogastroenterology. 2000;47:626-30. 
16. Hadad SM, Vaidya JS, Baker L, Koh HC, Heron TP, Hussain K, et al. Delay from symptom onset increases the conversion rate in laparoscopic cholecystectomy for acute cholecystitis. World J Surg. 2007;31:1298301.

17. González-Rodríguez FJ, Paredes-Cotoré JP, Pontón C, Rojo Y, Flores E, Luis-Calo ES, et al. Early or delayed laparoscopic cholecystectomy in acute cholecystitis? Conclusions of a controlled trial. Hepatogastroenterology. 2009;56:11-6.

18. Bhattacharya D, Senapati PS, Hurle R, Ammori BJ. Urgent versus interval laparoscopic cholecystectomy for acute cholecystitis: a comparative study. J Hepatobiliary Pancreat Surg. 2002:9:538-42.

19. Knight JS, Mercer SJ, Somers SS, Walters AM, Sadek SA, Toh SK. Timing of urgent laparoscopic cholecystectomy does not influence conversion rate. Br J Surg. 2004;91:601-4.

20. Greenwald JA, McMullen HF, Coppa GF, Newman RM. Standardization of surgeon-controlled variables: impact on outcome in patients with acute cholecystitis. Ann Surg. 2000;231:339-44.

21. Kortram K, Reinders JS, Ramshorst B, Wiezer MJ, Go PM, Boerma D. Laparoscopic cholecystectomy for acute cholecystitis should be performed by a laparoscopic surgeon. Surg Endosc. 2010;24:2206-9. 


\section{Colecistectomia por Laparoscopia e por Laparotomia na Colecistite Aguda: Análise Crítica de 520 Casos}

Acta Med Port 2014:27:685-691

Publicado pela Acta Médica Portuguesa, a Revista Científica da Ordem dos Médicos

Av. Almirante Gago Coutinho, 151

1749-084 Lisboa, Portugal.

Tel: +351218428215

E-mail: submissao@actamedicaportuguesa.com

www.actamedicaportuguesa.com

ISSN:0870-399X | e-ISSN: 1646-0758

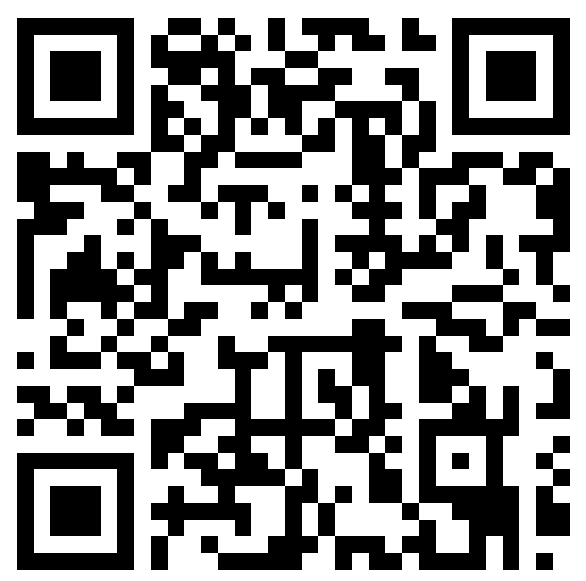

